Pacific Journal of Mathematics

HOMOGENEITY OF INFINITE PRODUCTS OF MANIFOLDS 


\title{
HOMOGENEITY OF INFINITE PRODUCTS OF MANIFOLDS WITH BOUNDARY
}

\author{
M. K. ForT, JR.
}

1. Introduction. In 1931, O. H. Keller [2] proved that the Hilbert cube $Q$ is homogeneous. V. L. Klee, Jr., proved [3] in 1955 that $Q$ is homogeneous with respect to finite sets, and in 1957 strengthened this result [4] by showing that $Q$ is homogeneous with respect to countable closed sets. Our Theorem 1 extends this latter result to spaces which are the product of a countably infinite number of manifolds with boundary. Our method of proof exploits the notion of category for the space of self-homeomorphisms of the product space, and differs considerably from the methods of Keller and Klee, who made use of convexity properties of linear spaces.

In Theorem 2 we prove that if $P$ is the product of a countably infinite number of manifolds with boundary and $U$ and $V$ are countable dense subsets of $P$, then there is a homeomorphism $h$ of $P$ onto itself such that $h[U]=V$. This theorem is analogous to a well known theorem about Euclidean spaces (see [1], p. 44). In a corollary to our Theorem 2, we show that if $U$ is a countable subset of the Hilbert cube $Q$, then there is a contraction $h_{t}, 0 \leqq t \leqq 1$, on $Q$ such that if $0<t<1$, then $h_{t}$ is a homeomorphism and $h_{t}[Q] \cap U=\phi$.

2. Notation and lemmas. For each positive integer $n$, we let $M_{n}$ be a compact manifold with boundary, and we let $B_{n}$ be the boundary of $M_{n}$. We let $P$ be the cartesian product space $M_{1} \times M_{2} \times M_{3} \times \cdots$. The projection mapping of $P$ into $M_{n}$ is denoted by $\pi_{n}$. If $x \in P$, we denote $\pi_{n}(x)$ by $x_{n}$. An admissible metric $d_{n}$ for $M_{n}$ is chosen so that $M_{n}$ has diameter less than $2^{-n}$, and we then define an admissible metric $d$ for $P$ by letting

$$
d(x, y)=\sum_{n=1}^{\infty} d_{n}\left(x_{n}, y_{n}\right)
$$

If $f$ and $g$ are mappings on a compact metric space $X$ into a metric space $Y$, we let $\rho(f, g)$ denote the least upper bound of the distances between $f(x)$ and $g(x)$ for $x$ in $X$.

The set of all homeomorphisms of $P$ onto $P$ is denoted by $H$. Although the metric space $(H, \rho)$ is not complete, it is topologically complete (i.e. homeomorphic to a complete metric space) and hence is

Received October 3, 1961. Written during a period in which the author was an Alfred P. Sloan Research Fellow. This work was also partially supported by National Science Foundation grant NSF-G12972. 
a second category space.

The following two lemmas can be proved using standard techniques, and the proofs are merely outlined.

LEMMA 1. If $M$ is a manifold with boundary $B, \alpha$ is an arc lying in $B, u$ and $v$ are the end points of $\alpha$, and $W$ is an open subset of $M$ which contains $\alpha$, then there is a homeomorphism \& of $M$ onto $M$ such that $\psi(u)=v$ and $\psi(x)=x$ for $x \in M-W$.

Proof. Let $S$ be the set of all points $t$ of $\alpha$ for which there exists a homeomorphism $\psi$ of $M$ onto $M$ such that $\psi(u)=t$ and $\psi(x)=x$ for $x \in M-W$. It is easy to see that $S$ is both open and closed relative to $\alpha$.

Lemma 2. If $M$ is a manifold with boundary $B$, the dimension of $M$ is at least 2, $C$ is a countable and compact subset of $M-B$, and $\phi$ is a homeomorphism on $C$ into $M-B$, then $\phi$ can be extended to $a$ homeomorphism $\Phi$ on $M$ onto $M$.

Proof. For each positive integer $n$, we can obtain compact sets $J_{n}$ and $K_{n}$ such that:

(i) $C$ is contained in the interior of $J_{n}$ and $\varphi[C]$ is contained in the interior of $K_{n}$;

(ii) each component of $J_{n}$ and of $K_{n}$ has diameter less than $1 / n$ and is homeomorphic to a spherical ball of dimension equal to that of $M$;

(iii) for each component $D$ of $J_{n}, \varphi[D \cap C]$ is contained in a single component of $K_{n}$; and

(iv) $J_{n} \supset J_{n+1}$ and $K_{n} \supset K_{n+1}$.

Using the sets $J_{n}$ and $K_{n}$, it is possible to construct homeomorphisms $\Phi_{n}$ of $M$ onto $M$ such that:

(i) if $D$ is a component of $J_{n}$ and $E$ is a component of $K_{n}$, then $\Phi_{n}[D] \subset E$ if and only if $\left.\left.\varphi\right] D \cap C\right] \subset E$; and

(ii) $\Phi_{n+1}(x)=\Phi_{n}(x)$ for all $x \in M-J_{n}$.

The sequence $\Phi_{1}, \Phi_{2}, \Phi_{3}, \cdots$ converges to the desired homeomorphism.

Lemma 3. If $p \in P$, there is a residual subset $R$ of $H$ such that if $h \in R$, then $h(p)_{n} \in M_{n}-B_{n}$ for each $n$.

Proof. Let $K_{n}=\left\{h \mid h \in H\right.$ and $\left.h(p)_{n} \in B_{n}\right\}$. It is obvious that each $K_{n}$ is closed. We want to prove that $K_{n}$ if nowhere dense. Thus, suppose $h \in K_{n}$ for some $n$ and that $\varepsilon>0$. We seek $g \in H-K_{n}$ such that $\rho(g, h)<\varepsilon$.

Choose an integer $m \neq n$ such that $M_{m}$ has diameter less than $\varepsilon$. We define $M=M_{n} \times M_{m}$. $\quad M$ is also a manifold with boundary, and the boundary $B$ of $M$ is the set $\left(M_{n} \times B_{m}\right) \cup\left(B_{n} \times M_{m}\right)$. Since $h \in K_{n}$, the point $\left(h(p)_{n}, h(p)_{m}\right)$ is a member of $B_{n} \times M_{m}$. Let $q$ be a point of $B_{m}$ 
such that $q \neq h(p)_{m}$. There is an arc $\beta$ in $B_{n} \times M_{m}$ which joins $\left(h(p)_{n}, h(p)_{m}\right)$ to $\left(h(p)_{n}, q\right)$ and has diameter less than $\varepsilon$ (since $M_{m}$ has diameter less than $\varepsilon$ ). We may now choose a point $r \in M_{n}-B_{n}$ and an arc $\gamma$ joining $(r, q)$ to $\left(h(p)_{n}, q\right)$ such that $\beta \cup \gamma$ is an arc and has diameter less than $\varepsilon$. We let $\alpha=\beta \cup \gamma$.

We now use Lemma 1 to obtain a homeomorphism $\psi$ of $M$ onto $M$ such that $\psi$ maps the point $\left(h(p)_{n}, h(p)_{m}\right)$ onto $(r, q)$ and the distance from $x$ to $\psi(x)$ is less than $\varepsilon$ for all $x \in M$.

Now, we define $g \in H$ by letting $g(y)_{k}=h(y)_{k}$ if $n \neq k \neq m$, and letting

$$
\left(g(y)_{n}, g(y)_{m}\right)=\psi\left(\left(h(y)_{n}, h(y)_{m}\right)\right) .
$$

Since $g(p)_{n}=r$ and $r \notin B_{n}, g \in H-K_{n}$. It is easy to see that $\rho(g, h)<\varepsilon$, and hence we have proved that $K_{n}$ is nowhere dense. We define $R=$ $H-\bigcup_{n=1}^{\infty} K_{n} . \quad R$ is a residual set and if $h \in R$, then $h(p)_{n} \notin B_{n}$ for all $n$.

Lemma 4. If $p$ and $q$ are points of $P$, then there is a residual subset $R$ of $H$ such that if $h \in R$, then $h(p)_{n} \neq h(q)_{n}$ for all $n$.

Proof. We define $J_{n}=\left\{h \mid h \in H\right.$ and $\left.h(p)_{n}=h(q)_{n}\right\}$. Each $J_{n}$ is closed. We want to prove that $J_{n}$ is nowhere dense. Suppose $h \in J_{n}$ and $\varepsilon>0$. We seek $g \in H-J_{n}$ such that $\rho(g, h)<\varepsilon$.

It follows from Lemma 3 , and the fact that residual subsets of $H$ are dense in $H$, that there exists $f \in H$ such that $\rho(f, h)<\varepsilon / 2$ and for all $k, f(p)_{k} \notin B_{k}$ and $f(q)_{k} \notin B_{k}$. If $f(p)_{n} \neq f(q)_{n}$ we can let $g=f$. Otherwise, we choose $m \neq n$ so that $f(p)_{m} \neq f(q)_{m}$ and define $M=M_{n} \times M_{m}$. Since $\left(f(p)_{n}, f(p)_{m}\right)$ and $\left(f(q)_{n}, f(q)_{m}\right)$ are not equal and neither is on the boundary of $M$, there is a homeomorphis $\varphi$ of $M$ onto $M$ such that the distance from $x$ to $\varphi(x)$ is less than $\varepsilon / 2$ for all $x \in M$ and such that the points $\varphi\left(\left(f(p)_{n}, f(p)_{m}\right)\right)$ and $\varphi\left(\left(f(q)_{n}, f(q)_{m}\right)\right)$ have different first coordinates. We now define $g \in H$ by letting $g(y)_{k}=f(y)_{k}$ if $n \neq k \neq m$, and $\left(g(y)_{n}, f(y)_{m}\right)=\varphi\left(\left(f(y)_{n}, f(y)_{m}\right)\right)$. It is easy to see that $\rho(g, f)<\varepsilon / 2$ and hence $\rho(g, h)<\varepsilon$. Moreover, $g(p)_{n} \neq g(q)_{n}$ and hence $g \in H-J_{n}$.

We obtain the desired residual set $R$ by letting $R=H-\bigcup_{n=1}^{\infty} J_{n}$.

THEOREM 1. If $A$ is a closed and countable subset of $P$ and $f$ is a homeomorphism on $A$ into $P$, then $f$ can be extended to a homeomorphism $F$ on $P$ onto $P$.

Proof. There is no loss in generality in assuming that each $M_{n}$ has dimension at least 2 , for otherwise we could define $S_{n}=M_{2 n-1} \times M_{2 n}$ and represent $P$ as $S_{1} \times S_{2} \times S_{3} \times \cdots$.

It follows from Lemma 3 and Lemma 4 that there is a homeomorphism 
$h \in H$ such that for each $n$, the projection mapping $\pi_{n}$ maps both $h[A]$ and $h f[A]$ in a one-to-one manner into $M_{n}-B_{n}$. The mapping $\varphi_{n}=$ $\pi_{n} h f h^{-1} \pi_{n}^{-1}$ is one-to-one on $\pi_{n} h[A]$ onto $\pi_{n} h f[A]$ and can be extended by Lemma 2 to a homeomorphism $\Phi_{n}$ on $M_{n}$ onto $M_{n}$. We obtain $\Phi \in H$ by letting $\Phi(x)_{n}=\Phi_{n}\left(x_{n}\right)$. The desired extension $F$ of $f$ is obtained by defining $F=h^{-1} \Phi h$.

Let $h$ be a homeomorphism on a compact space $X$ into a compact space $Y$, and let $n$ be a positive integer. We define

$$
\eta(h, n)=2^{-n} \cdot \inf \{d(h(x), h(y)) \mid x, y \in X \text { and } d(x, y) \geqq 1 / n\} .
$$

Lemma 5. If $h_{1}, h_{2}, h_{3}, \cdots$ is a sequence of homeomorphisms on $X$ onto $Y$ such that $\rho\left(h_{n}, h_{n+1}\right)<\eta\left(h_{n}, n\right)$, then the sequence converges uniformly to a homeomorphism $h$ on $X$ into $Y$.

Proof. It is clear that the sequence converges uniformly to a continuous function $h$ on $X$ into $Y$. We must prove that $h$ is one-to-one.

Suppose $u$ and $v$ are distinct points of $X$. We choose $n>1$ so that $d(u, v)>1 / n$. Then, for $k \geqq n$,

$$
\begin{aligned}
d\left(h_{k+1}(u), h_{k+1}(v)\right) & \geqq d\left(h_{k}(u), h_{k}(v)\right)-d\left(h_{k}(u), h_{k+1}(u)\right)-d\left(h_{k}(v), h_{k+1}(v)\right) \\
& \geqq d\left(h_{k}(u), h_{k}(v)\right)-2 \eta\left(h_{k}, k\right) \\
& \geqq d\left(h_{k}(u), h_{k}(v)\right)-2 \cdot 2^{-k} d\left(h_{k}(u), h_{k}(v)\right) \\
& \geqq d\left(h_{k}(u), h_{k}(v)\right) \cdot\left(1-2^{-k+1}\right) .
\end{aligned}
$$

Thus,

$$
\begin{aligned}
d(h(u), h(v)) & =\lim _{k \rightarrow \infty} d\left(h_{k}(u), h_{k}(v)\right) \\
& \geqq d\left(h_{n}(u), h_{n}(v)\right) \cdot \prod_{j=n}^{\infty}\left(1-2^{-j+1}\right) \\
& \geqq d\left(h_{n}(u), h_{n}(v)\right) / 4, \quad(\text { since } n>1) .
\end{aligned}
$$

This proves that $h$ is one-to-one and hence a homeomorphism.

THEOREM 2. If $U$ and $V$ are countable dense subsets of $P$, then there is a homeomorphism $h$ of $P$ onto $P$ such that $h[U]=V$.

Proof. As we have remarked in the proof of Theorem 1, there is no loss in generality in assuming that each $M_{n}$ has dimension at least 2. In view of Lemma 3 and Lemma 4, we may also assume that $U$ and $V$ are so situated in $P$ that each $\pi_{n}$ maps both $U$ and $V$ in a one-to-one manner into $M_{n}-B_{n}$.

We are going to arrange the points of $U$ and $V$ into sequences $u_{1}, u_{2}, u_{3}, \cdots$ and $v_{1}, v_{2}, v_{3}, \cdots$ and choose homeomorphisms $h_{i j}$ for all 
positive integers $i$ and $j$. This is done by a fairly complicated inductive process, the first four steps of which are given below. We let $U_{1}=U$, $V_{1}=V$, and as soon as $u_{1}, \cdots, u_{n}$ and $v_{1}, \cdots, v_{n}$ are defined, we let $U_{n+1}=U_{n}-\left\{u_{1}, \cdots, u_{n}\right\}, V_{n+1}=V_{n}-\left\{v_{1}, \cdots, v_{n}\right\}$. We assume that $U$ and $V$ are well ordered so as to have the order type of the positive integers. We let $H_{n}$ be the set of homeomorphisms of $M_{n}$ onto itself.

Step 1. $u_{1}$ is chosen to be the first point of $U$ and $v_{1}$ is chosen to be the first point of $V . h_{11} \in H_{1}$ is chosen so that $h_{11} \pi_{1}\left(u_{1}\right)=\pi_{1}\left(v_{1}\right)$. $h_{1 j} \in H_{j}$ is the identity for $j>1$.

Step 2. $v_{2}$ is the first point of $V_{2} . \quad u_{2} \in U_{2}$ is chosen near enough to $v_{2}$ for us to obtain $h_{21} \in H_{1}$ so that: $\rho\left(h_{21}, h_{11}\right)<\eta\left(h_{11}, 1\right)$ and $h_{21} \pi_{1}\left(u_{j}\right)=$ $\pi_{1}\left(v_{j}\right)$ for $j=1,2$. $h_{22} \in H_{2}$ is chosen so that $h_{22} \pi_{2}\left(u_{j}\right)=\pi_{2}\left(v_{j}\right)$ for $j=1,2$. $h_{2 j} \in H_{j}$ is the identity for $j>2$.

Step 3. $u_{3}$ is the first point of $U_{3} . v_{3} \in V_{3}$ is chosen near enough to $u_{3}$ for us to obtain $h_{3 i} \in H_{i}$ so that: $\rho\left(h_{3 i}, h_{2 i}\right)<\eta\left(h_{2 i}, 2\right)$ and $h_{3 i} \pi_{i}\left(u_{j}\right)=$ $\pi_{i}\left(v_{j}\right)$ for $i=1,2$ and $j=1,2,3 . \quad h_{33} \in H_{3}$ is chosen so that $h_{33} \pi_{3}\left(u_{j}\right)=$ $\pi_{3}\left(v_{j}\right)$ for $j=1,2,3$. $h_{3 j} \in H_{j}$ is the identity for $j>3$.

Step 4. $v_{4}$ is the first point of $V_{4} \cdot u_{4} \in U_{4}$ is chosen near enough to $v_{4}$ for us to obtain $h_{4 i} \in H_{i}$ so that: $\rho\left(h_{4 i}, h_{3 i}\right)<\eta\left(h_{3 i}, 3\right)$ and $h_{4 i} \pi_{i}\left(u_{j}\right)=$ $\pi_{i}\left(v_{j}\right)$ for $i=1,2,3$ and $j=1, \cdots, 4 . \quad h_{44} \in H_{4}$ is chosen so that $h_{44} \pi_{4}\left(u_{j}\right)=$ $\pi_{4}\left(v_{j}\right)$ for $j=1, \cdots, 4$. $h_{4 j} \in H_{j}$ is the identity for $j>4$.

We continue this process. By Lemma 5, the homeomorphisms $h_{j 1}, h_{j 2}, h_{j 3}, \cdots$ converge uniformly to a homeomorphism $g_{j} \in H_{j}$. It is easy to see that $g_{j} \pi_{j}\left(u_{i}\right)=\pi_{j}\left(v_{i}\right)$ for all $i$ and $j$. There is determined uniquely a homeomorphism $h \in H$ for which $\pi_{j} h=g_{j} \pi_{j}$ for all $j$. Since $h\left(u_{i}\right)=v_{i}$ for all $i$, and $U=\left\{u_{1}, u_{2}, \cdots\right\}, V=\left\{v_{1}, v_{2}, \cdots\right\}, h$ is the desired homeomorphism.

CoRollary. If $C$ is a countable subset of the Hilbert cube $Q$, then there is a contraction $h_{t}, 0 \leqq t \leqq 1$, defined on $Q$ such that:

(i) $h_{1}$ is the identity,

(ii) $h_{0}$ is a constant mapping, and

(iii) if $0<t<1, h_{t}$ is a homeomorphism of $Q$ into $Q$ and $h_{t}[Q] \cap C=\phi$.

Proof. We let $M_{n}$ be the closed interval $\left[-5^{-n}, 5^{-n}\right]$. The resulting space $P$ may then be thought of as the Hilbert cube $Q$. (This representation is used since $M_{n}$ was assumed to have diameter less than $2^{-n}$.) We let $D$ be the set of all points $x$ in $P$ such that $\pi_{i}(x)$ is rational for 
all $i$, and $\pi_{i}(x)=5^{-i}$ for all but a finite number of values of $i$ :

Both $C \cup D$ and $D$ are countable and dense in $P$, so by Theorem 2 there is a homeomorphism $G$ of $P$ onto $P$ such that $G[C \cup D]=D$. We define $g_{t}(x)=t x$ for $0 \leqq t \leqq 1$ and $x \in P$. Finally, we let $h_{t}=$ $G^{-1} g_{t} G$. It is easy to see that the desired contraction is $h_{t}, 0 \leqq t \leqq 1$.

\section{REFERENCES}

1. W. Hurewicz and H. Wallman, Dimension Theory, Princeton 1941.

2. Ott-Heinrich Keller, Die Homoiomorphie der kompakten konvexen Mengen im Hilbertschen Raum, Math. Ann., 105 (1931), 748-758.

3. V. L. Klee, Jr., Some topological properties of convex sets, Trans. Amer. Math. Soc., 78 (1955), 30-45.

4. - Homogeneity of infinite-dimensional parallelotopes, Annals of Math., 66 (1957), 454-460.

UNIVERSITY OF GEORGIA 


\title{
PACIFIC JOURNAL OF MATHEMATICS
}

\author{
EDITORS
}

RalPh S. Phillips

Stanford University

Stanford, California

M. G. Arsove

University of Washington

Seattle 5 , Washington
A. L. Whiteman

University of Southern California Los Angeles 7, California

LOWell J. PAIGE

Unıversity of California

Los Angeles 24, California

\section{ASSOCIATE EDITORS}
E. F. BECKENBACH
D. DERRY
H. L. ROYDEN
E. G. STRAUS
T. M. CHERRY
M. OHTSUKA
E. SPANIER
F. WOLF

\section{SUPPORTING INSTITUTIONS}

\author{
UNIVERSITY OF BRITISH COLUMBIA \\ CALIFORNIA INSTITUTE OF TECHNOLOGY \\ UNIVERSITY OF CALIFORNIA \\ MONTANA STATE UNIVERSITY \\ UNIVERSITY OF NEVADA \\ NEW MEXICO STATE UNIVERSITY \\ OREGON STATE UNIVERSITY \\ UNIVERSITY OF OREGON \\ OSAKA UNIVERSITY \\ UNIVERSITY OF SOUTHERN CALIFORNIA
}

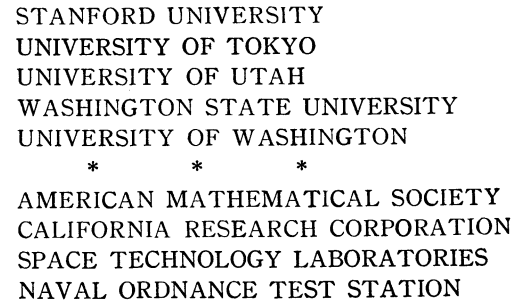

Mathematical papers intended for publication in the Pacific Journal of Mathematıcs should be typewritten (double spaced), and the author should keep a complete copy. Manuscripts may be sent to any one of the four editors. All other communications to the editors should be addressed to the managing editor, L. J. Paige at the University of California, Los Angeles 24, California.

50 reprints per author of each article are furnıshed free of charge; additional copies may be obtained at cost in multiples of 50 .

The Pacific Journal of Mathematics is published quarterly, in March, June, September, and December. Effective with Volume 13 the price per volume (4 numbers) is $\$ 18.00$; single issues, $\$ 5.00$. Special price for current issues to individual faculty members of supporting institutions and to individual members of the American Mathematical Society: $\$ 8.00$ per volume; single issues $\$ 2.50$. Back numbers are available.

Subscriptions, orders for back numbers, and changes of address should be sent to Pacific Journal of Mathematics, 103 Highland Boulevard, Berkeley 8, California.

Printed at Kokusai Bunken Insatsusha (International Academic Printing Co., Ltd.), No. 6 , 2-chome, Fujimi-cho, Chiyoda-ku, Tokyo, Japan.

PUBLISHED BY PACIFIC JOURNAL OF MATHEMATICS, A NON-PROFIT CORPORATION

The Supporting Institutions listed above contribute to the cost of publication of this Journal, but they are not owners or publishers and have no responsibility for its content or policies. 


\section{Pacific Journal of Mathematics}

\section{Vol. 12, No. $3 \quad$ March, 1962}

Alfred Aeppli, Some exact sequences in cohomology theory for Kähler

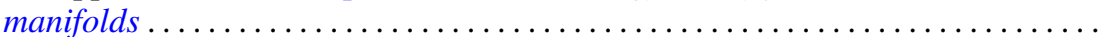

Paul Richard Beesack, On the Green's function of an N-point boundary value

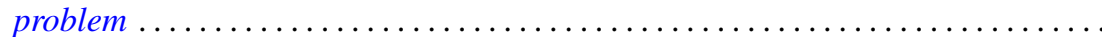

James Robert Boen, On p-automorphic p-groups....

James Robert Boen, Oscar S. Rothaus and John Griggs Thompson, Further results

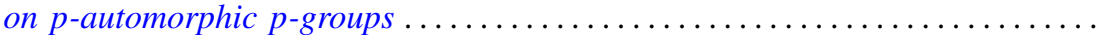

James Henry Bramble and Lawrence Edward Payne, Bounds in the Neumann problem for second order uniformly elliptic operators ..................

Chen Chung Chang and H. Jerome (Howard) Keisler, Applications of ultraproducts of pairs of cardinals to the theory of models .........................

Stephen Urban Chase, On direct sums and products of modules ................

Paul Civin, Annihilators in the second conjugate algebra of a group algebra .......

J. H. Curtiss, Polynomial interpolation in points equidistributed on the unit

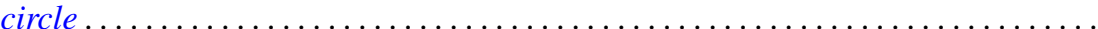

Marion K. Fort, Jr., Homogeneity of infinite products of manifolds with

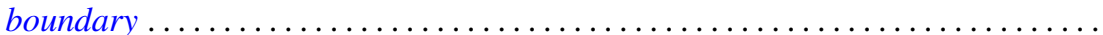

James G. Glimm, Families of induced representations . . . . . . . . . . . . . . .

Daniel E. Gorenstein, Reuben Sandler and William H. Mills, On almost-commuting

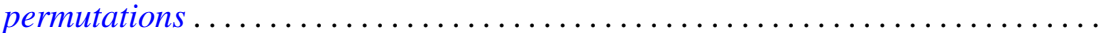

Vincent C. Harris and M. V. Subba Rao, Congruence properties of $\sigma_{r}(N) \ldots \ldots \ldots$

Harry Hochstadt, Fourier series with linearly dependent coefficients . . . . . . . . . . 925

Kenneth Myron Hoffman and John Wermer, A characterization of $C(X)$. .

Robert Weldon Hunt, The behavior of solutions of ordinary, self-adjoint differential equations of arbitrary even order...

Edward Takashi Kobayashi, A remark on the Nijenhuis tensor

David London, On the zeros of the solutions of $w^{\prime \prime}(z)+p(z) w(z)=0$

Gerald R. Mac Lane and Frank Beall Ryan, On the radial limits of Blaschke products...

T. M. MacRobert, Evaluation of an E-function when three of its upper parameters

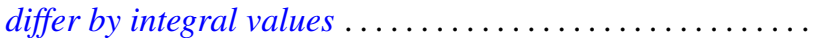

Robert W. McKelvey, The spectra of minimal self-adjoint extensions of a symmetric operator

Adegoke Olubummo, Operators of finite rank in a reflexive Banach space. .

David Alexander Pope, On the approximation of function spaces in the calculus of variations

Bernard W. Roos and Ward C. Sangren, Three spectral theorems for a pair of singular first-order differential equations...............

Arthur Argyle Sagle, Simple Malcev algebras over fields of characteristic zero .

S. C. Tang, Some theorems on the ratio of empirical distribution to the theoretical distribution

Robert Charles Thompson, Normal matrices and the normal basis in abelian number fields.

Howard Gregory Tucker, Absolute continuity of infinitely divisible distributions ... 